\title{
Erratum: Translation constraints on quantum phases with twisted boundary conditions [Phys. Rev. B 100, 174435 (2019)]
}

\author{
Shunsuke C. Furuya $\odot$ and Yusuke Horinouchi
}

(Received 12 July 2020; published 28 July 2020)

DOI: 10.1103/PhysRevB.102.019902

We correct errors of citation in the original article.

(i) P. 1: The sentence "Yao and Oshikawa reported quite recently a paper that follows this line [11]" cites a paper [11] by Y. Yao, C.-T. Hsieh, and M. Oshikawa. This sentence should cite another paper by Y. Yao and M. Oshikawa [1]. The same mistakes of citation are found in the original article as shown below.

(ii) P. 2, the first paragraph: The sentence "In that context, we discuss the LSM-type ingappability on the checkerboard lattice as a continuation of the work in Refs. [11,12]" should cite Refs. [1,2].

(iii) P. 3, above Eq. (11): The sentence "Let us impose the tilted boundary condition on the system [11]" should cite Ref. [1] instead of [11] of the original article.

(iv) P. 6, the first paragraph of Sec. VII: The citation of Ref. [11] in the sentence "Nevertheless, the well-known flux insertion argument turned out not to demonstrate the anomaly explicitly in the periodic [3] or the tilted [11] boundary conditions" should be replaced as Ref. [1].

(v) P. 7, the final paragraph: The sentence "Just as Ref. [11] did in the tilted boundary condition, we can extend ..." should cite Ref. [1] instead of Ref. [11] of the original article.

[1] Y. Yao and M. Oshikawa, Generalized Boundary Condition Applied to Lieb-Schultz-Mattis-Type Ingappabilities and ManyBody Chern Numbers, Phys. Rev. X 10, 031008 (2020).
[2] S. C. Furuya and M. Oshikawa, Symmetry Protection of Critical Phases and a Global Anomaly in $1+1$ Dimensions, Phys. Rev. Lett. 118, 021601 (2017). 